\title{
Astrochemistry on the EXPOSE/ISS and BIOPAN/Foton experiments
}

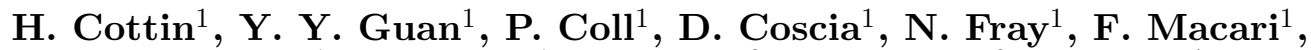 \\ F. Stalport ${ }^{1}$, F. Raulin ${ }^{1}$, C. Szopa ${ }^{2}$, D. Chaput ${ }^{3}$, M. Viso ${ }^{4}$, \\ M. Bertrand ${ }^{5}$, A. Chabin ${ }^{5}$, F. Westall ${ }^{5}$ and A. Brack \\ ${ }^{1}$ LISA (Univ. Paris 7 \& Paris 12, CNRS), av. du G ${ }^{\text {al }}$ de Gaulle, 94010 Créteil Cedex, France \\ email: herve.cottin@lisa.univ-paris12.fr \\ ${ }^{2}$ LATMOS-IPSL (UPMC, UVSQ, CNRS), 4 place Jussieu, 75005 Paris Cedex, France \\ ${ }^{3}$ CNES, Centre Spatial de Toulouse, 18 avenue Edouard Belin, 31401 Toulouse Cedex 9, France \\ ${ }^{4}$ CNES, 2 place Maurice Quentin, 75039 Paris Cedex 01, France \\ ${ }^{5} \mathrm{CBM}$ (CNRS), rue Charles-Sadron, 45071 Orléans Cedex 2, France
}

\begin{abstract}
We describe three space experiments designed to expose to space conditions, and more specifically to solar UV radiation, selected samples of organic and mineral material.
\end{abstract}

Solar UV radiation is a major source of energy for initiating chemical evolution towards complex organic structures but it can also photodissociate the most elaborate molecules. Thus, Solar UV can erase the organic traces of past life on the surface of planets, such as Mars (Oro \& Holzer 1979), destroy organic molecules present on meteorites, Barbier et al. 1998, influence the production of distributed sources in comets (Cottin et al. 2004) or initiate chemistry in Titan's atmosphere (Sagan \& Thompson 1984). In the interstellar medium, the UV radiation field emitted by stars in the galaxy is also responsible for the chemical evolution and the extraordinary diversity of detected organic molecules.

To improve our knowledge of the chemical nature and evolution of organic molecules involved in extraterrestrial environments with astrobiological implications, we have developed a series of three space experiments implemented on the Russian automated capsule FOTON, or outside of the International Space Station. The goal of these experiments is to expose to space conditions, and more specifically to solar UV radiation, selected samples of organic and mineral material. The UVolution experiment was flown in September 2007, during 12 days, in the ESA BIOPAN facility set outside the FOTON M3 capsule (Demets, Schulte \& Baglioni 2005). The PROCESS experiment has been installed on the ISS EXPOSE-Eutef facility from February 2008 to August 2009, and the AMINO experiment is currently on the ISS EXPOSE-R facility and will be returned to ground after 1.5 years of exposure to space conditions (Rabbow et al. 2009).

Most of the previous astrochemistry experiments implemented in space so far were carried out in open cells exposed to solar UV radiation (Barbier et al. 1998, Barbier et al. 2002, Boillot et al. 2002). In these types of experiments, solid organic samples are deposited behind a UV-transparent window and exposed to the flux of solar radiation. If the studied molecule is sensitive to energetic photons, its photodestruction can be quantified when the sample are returned to Earth for analysis. However, gaseous products resulting from photolysis are lost to space. A first use of closed cells was reported in (Ehrenfreund et al. 2007), while a more advanced concept is presented in (Cottin et al. 2008). Such sealed cells allow study of the chemical evolution in the gaseous phase, as well as investigation of heterogeneous processes, such as the degradation of solid compounds and the release of gaseous fragments. 


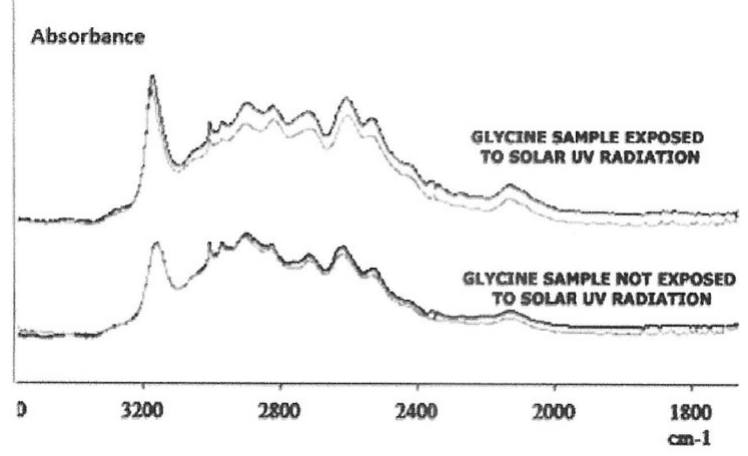

Figure 1. Infrared spectra of two open cells containing glycine before and after exposition in space during UVolution. The two top spectra show measurements for a cell exposed to solar UV (variation=-11\%), while the two spectra at the bottom show measurements for a flight control cell (not exposed to solar UV, variation=0\%). From the integrated exposition time of samples to UV (29 hrs), we estimate a half-lifetime of 8 days $( \pm 10 \%)$ for glycine at $1 \mathrm{AU}$.

Samples returned to Earth after the UVolution experiment have been analyzed in the laboratory. After processing of the measurements, the photochemical lifetime of the molecule at $1 \mathrm{AU}$ is calculated, and can be extrapolated at other heliocentric distances and other astrophysical environments (diffuse interstellar medium, dark clouds).

Figure 1 shows an example of data processing in the case of glycine using infrared measurements. The measurements are also compared to experimental simulations performed in the laboratory on the same kind of samples with usual VUV lamps. The ratio space/lab result depends on the molecules and can be as high as a few hundreds, showing that laboratory VUV lamps are rather poor simulators of the solar flux.

The first use of closed cells has been successful. The analysis of an exposed mixture of $\mathrm{CH}_{4} / \mathrm{N}_{2}$ resulted as expected in the formation of $\mathrm{C}_{2} \mathrm{H}_{6}$ and $\mathrm{C}_{2} \mathrm{H}_{4}$ which were detected by gas chromatography. Complete results of the UVolution experiment will be published soon (Guan et al. 2009, in prep., Stalport et al. 2009, Stalport et al. 2009, in prep.).

\section{References}

Barbier, B., Chabin, A., Chaput, D., \& Brack, A. 1998, Planet. Space Sci. 46, 391

Barbier, B., Henin, O., Boillot, F., Chabin, A., Chaput, D., \& Brack, A. 2002, Planet. Space Sci. 50,353

Boillot, F., Chabin, A., Buré, C., Venet, M., Belsky, A., Bertrand-Urbaniak, M., Delmas, A., Brack, A., \& Barbier, B. 2002, Orig. Life Evol. Biosph. 32, 359

Cottin, H., B'enilan, Y., Gazeau, M.-C., \& Raulin, F. 2004, Icarus 167, 397

Cottin, H., Coll, P., Coscia, D., Fray, N., Guan, Y. Y., Macari, F., Raulin, F., Rivron, C., Stalport, F., Szopa, C., Chaput, D., Viso, M., Bertrand, M., Chabin, A., Thirkell, L., Westall, F., \& Brack, A. 2008, Adv. Space Res. 42, 2019

Demets, R., Schulte, W., \& Baglioni, P. 2005, Adv. Space Res. 36, 311

Ehrenfreund, P., Ruiterkamp, R., Peeters, Z., Foing, B., Salama, F., \& Martins, Z. 2007, Planet. Space Sci. 55, 383

Oro, J. \& Holzer, G. 1979, Journal of Molecular Evolution 14, 153

Rabbow, E., Horneck, G., Rettberg, P., Schott, J.-U., Panitz, C., L'Afflitto, A., von HeiseRotenburg, R., Willnecker, R., Baglioni, P., Hatton, J., Dettmann, J., Demets, R., \& Reitz, G. 2009, Orig. Life Evol. Biosph., in press (DOI 10.1007/s11084-009-9173-6)

Sagan, C. \& Thompson, W. R. 1984, Icarus 59, 133

Stalport, F., Guan, Y. Y., Coll, P., Szopa, C., Macari, F., Raulin, F., \& Cottin, H. 2009, Astrobiology, submitted 